\title{
Experiments and Simulations on Coastal Flows in the Presence of a Topographic Slope
}

\author{
L. ZAVAla SANSÓN \\ Department of Physical Oceanography, CICESE, Ensenada, Baja California, México \\ R. SERRAVALL \\ Università degli Studi di Modena e Reggio Emilia, Modena, Italy \\ G. F. CARnevale \\ Scripps Institution of Oceanography, San Diego, California \\ G. J. F. VAN HEIJST \\ Eindhoven University of Technology, Eindhoven, Netherlands
}

(Manuscript received 13 March 2004, in final form 19 April 2005)

\begin{abstract}
The evolution of a barotropic coastal current in the presence of a bottom ramp-shaped topography is studied by means of laboratory experiments and numerical simulations. The experiments are performed in a rectangular rotating tank filled with freshwater. The fluid depth is shallow at one side of the domain and deeper at the other side, and both regions are divided by a narrow slope, whose depth contours are perpendicular to the long sides of the tank. A current approaching the slope is produced along one of the vertical walls, having the boundary at its right. Two configurations are analyzed: when the current flows from shallow to deep water and when flowing in the opposite direction. In the first scenario, the current is divided in two parts, one of them following the coastline. The other part of the current pairs with a cell of negative relative vorticity generated at the slope due to squeezing effects, forming a dipolar structure moving offshore, back toward the shallow side. In addition, a weak current moving inshore along the slope is clearly formed. In the second configuration, when the flow goes from deep to shallow water, a part of or even the whole current might be forced to move along the contours of the topography, away from the coast. In this case there is no dipole formation. The experiments are well reproduced by means of quasigeostrophic numerical simulations, which allow a more detailed systematic study of the influence of flow parameters such as the topography height and the width of the slope.
\end{abstract}

\section{Introduction}

This paper addresses the evolution of a coastal current in the presence of an underwater escarpment running perpendicular to the coast. The main goal is to understand the dynamics of the current when it crosses this topographic feature and its dependence on the direction of the flow, which can be from shallow to deep water or in the opposite direction. For instance, the presence of the topography leads to the bifurcation of

Corresponding author address: Luis Zavala Sansón, Department of Physical Oceanography, CICESE, P.O. Box 434844, San Diego, CA 92143-4844.

E-mail: lzavala@cicese.mx the current, which may be detached from the coast and flow along the escarpment, while creating more complicated structures. To gain a clear physical understanding of the problem, the current-topography interaction is examined by means of idealized laboratory experiments in a rotating tank and by numerical simulations. The laboratory experiments provide robust, physical evidence of the typical behavior of the coastal flow due to topographic variations. The numerical part is based on the conventional quasigeostrophic formulation, and constitutes a suitable oceanographical model of coastal currents.

The study is motivated by numerous geophysical situations where bottom topography may play an important role in the evolution of coastal currents, subject to 
the earth's rotation. The bifurcation of boundary currents forming long filaments along a perpendicular topographic feature has been observed, for instance, in the Adriatic Sea where the current flowing along the coast of Albania bifurcates when approaching the Jabuka Pit (Poulain 1999). A similar behavior is described for the Sicily Channel by Serravall (1999). Other examples are the Bering slope current (Kinder et al. 1986) and the flux along the Iceland-Faroe Ridge (Hansen and Meincke 1979).

The deflection of a coastal current by an underwater escarpment running perpendicular to the coast has been addressed in a number of fundamental papers. The problem is particularly intriguing because of the somewhat surprising asymmetrical behavior of the flow. Recently, additional interest has been generated by progress in the nonlinear theory of the problem with results contrasting considerably from the linear theoretical predictions.

Linear shallow water theory shows that a current flowing toward a step will be deflected along the step in the direction with the shallow fluid on the right (in the Northern Hemisphere). This is independent of whether the initial flow approaches the step from the shallow or deep side (Gill et al. 1986; Willmott and Grimshaw 1991; Johnson and Davey 1990) and is related to the fact that the double Kelvin wave that forms over the step propagates unidirectionally with shallow water on the right. Thus, if there is a coastal boundary with a perpendicular step, linear theory would predict the current to either be pushed toward the coast or deflected away from it depending on whether there is shallow fluid lying on the left or right of the step when viewed from the coast, and the sense of the deflection would be independent of the initial direction of the coastal current. For convenience, we shall refer to the configuration where the shallow water is on the left (right) as one looks offshore from the coast along the step, as the left (right)-handed geometry [hereinafter left (right) geometry]. The predictions-at least for the initial tendencies - of the linear theory were verified in laboratory experiments by Gill et al. (1986) and Allen (1996) in which a water surface height disturbance running along the shore is allowed to relax in a classic Rossby wave adjustment problem.

The long time adjustment problem in the shallow water theory presents some peculiar results that force one to consider the full nonlinear problem. The first signature of difficulty is that in the linear theory there is the formation of a singularity in the flow at the coast in the left geometry, that is, the case where the current tries to approach the coast (Johnson 1985; Johnson and Davey 1990). Furthermore, it is found that, in the sta- tionary state, there is no flow across the step and the flow on each side of the step is in opposite directions (Gill et al. 1986). This result of linear theory seems somewhat unrealistic. If we consider real coastal flows, then we would reasonably expect that small cross-shore topographic perturbations cause little deflection, while for an escarpment with sufficiently large topographic variation we might imagine that the streamlines follow the topographic contours. This separation imposed by any step in the linear problem is not ameliorated by the broadening of the step or, in other words, making the slope finite. In fact, it can be shown that in the linear theory there can be no stationary flow anywhere on the slope (Willmott and Grimshaw 1991; Allen 1996). Even the broadened step or escarpment acts as a complete barrier to stationary flow in linear theory, and in the left geometry the singularity at the wall is still present. The noncommunication across the topography and the singularity along the coast are both unrealistic artifacts of the dropping of the advection terms in the linearized equations of motion. Although adding viscosity to the problem can allow communication between the basins and eliminate the singularity at the coast, this is an artifice that avoids the essential difficulty caused by the lack of nonlinear advection.

The effects of nonlinear advection in flow over an escarpment were considered by Allen (1988), Spitz and Nof (1991), and Stern and Austin (1995). In these cases, there can be continuous flow across and along the escarpment with no barrier to the necessary entrainment of still waters that allow for smooth solutions. These articles present analytical results based on the integral properties of the flow, that is, based on the Bernoulli equation and the conservation of potential vorticity. The analytical strategy used avoids the complicated details in the region where the escarpment intersects the coast to deduce far-field properties of the flow. Laboratory experiments in a rotating tank were performed to confirm the theoretical predictions. For the infinite slope case, Spitz and Nof (1991) found that, indeed, for the right geometry a current along the wall did turn to follow the step as predicted. In the left geometry several experiments were performed. In one case, strong eddying motions were noted with no approach to steady state; another experiment showed the current moving at an angle to the step, while some other experiments did show an offshore current following the escarpment. Stern and Austin (1995) performed laboratory experiments in the left geometry for the finite slope case. Unexpectedly, although a branch of the current did leave the boundary, it did not flow along the slope, but formed a dipole that moved away from the 
topography, back into the region of the source of the boundary current.

In Carnevale et al. (1999, hereinafter C99) another analytic approach was taken. In an attempt to understand the failure of the predictions based on linear shallow water theory, and to go beyond the approach based solely on integral invariants, a complete analytic solution was obtained. This was possible by working with quasigeostrophic theory (QG). Although this is limited to small topographic variations, it did maintain the important aspect of the effects of nonlinear advection lost in the linearized shallow water theory. If the QG theory is linearized, that is, if the nonlinear advection terms are dropped, then the stationary solutions are similar to that of the linear shallow water theory with no flow on the slope and flow in opposite direction on opposite sides of the escarpment. However, the results are very different with the inclusion of the nonlinear advection terms. By assuming the upstream coastal current had an exponential profile, it proved possible to reduce the Green's function for the problem to a one-dimensional integral, making the full solution for an escarpment of arbitrary topography easily calculable. This solution showed clearly that in the right-handed geometry the stationary solution has an outward flowing current along the contours of the topography. Surprisingly, for the left geometry the QG solution shows an inward flowing current along the contours of the topography. C99 performed QG numerical simulations in which a coastal jet encounters an escarpment and demonstrated that the same effect of dipole formation for the left geometry observed by Stern and Austin (1995) also occurs in the QG problem in detail. So the effect is captured by the QG theory, and there is no necessity to invoke shallow water effects to investigate it.

Here we demonstrate in a laboratory experiment similar to Stern and Austin's that the inshore current suggested in other studies is created over the topography. It will be shown that it is initiated early in the production of the separating dipole and starts near the coast, and slowly gains strength farther out along the escarpment. Furthermore, we show that the production of this dipole in the left geometry occurs repeatedly, perhaps preventing a steady state from being reached. It will be illustrated that the point of separation of the free dipole from the escarpment is farther from the coast the steeper the topography, so it appears possible that separation may not occur in a sufficiently narrow body of water. Last, an example will be shown in the right geometry where the flow bifurcates at the escarpment with part of it following the slope as expected (C99). This process occurs despite the complicated long- term structure of the experimental current. Strangely, Stern and Austin (1995) did not attempt to see this current bifurcation in the right geometry.

The paper is organized as follows: in section 2 the laboratory experiments are described. Section 3 contains QG numerical simulations for different flow parameters. An additional numerical analysis with passive tracers is shown in section 4 , and the conclusions are presented in section 5 .

\section{Laboratory experiments}

\section{a. Experimental setup}

The laboratory experiments are performed in a rectangular rotating tank with horizontal dimensions 150 $\mathrm{cm} \times 100 \mathrm{~cm}$. The tank is filled with fresh tap water and rotates in counterclockwise direction at a constant rate $\Omega$ (with an associated Coriolis parameter $f=2 \Omega$ ). The experimental procedure consisted of setting the tank in rotation at the specified constant angular speed about 30 minutes before the start of an experiment in order to ensure that the fluid has reached a state of solid-body rotation.

The bottom topography of the tank is shallow at one side and deeper at the opposite part, both regions being divided by a narrow slope as shown in Fig. 1. The deep side of the tank has a depth $H_{0}$. On the shallow side the bottom is raised to a height $\delta H$ above the deep side producing a depth $H_{0}-\delta H$. The slope has a width $W_{0}$. Taking the downslope direction as the $x$ axis, with the origin at the edge of the shallow side, the fluid depth is given by

$$
h(x)=\left\{\begin{array}{cc}
H_{0}-\delta H, & x<0 \\
H_{0}-\delta H+\frac{\delta H}{W_{0}} x, & 0 \leq x \leq W_{0} \\
H_{0}, & x>W_{0} .
\end{array}\right.
$$

A current parallel to the long side of the tank is created, pointing toward the topography. Two types of experiments were performed: coastal currents moving from shallow to deep and from deep to shallow water, in both situations having the wall to the right. These cases correspond with the left- and right-handed geometries defined by C99, respectively. The other two possibilities (coastal flows with the wall to the left) were not considered. In such cases, the current quickly detaches from the wall, obscuring the essential problem to be analyzed here.

The method for creating the current consisted of injecting fluid at a constant rate by means of a thin tube (3-cm diameter) vertically immersed in the tank. The 


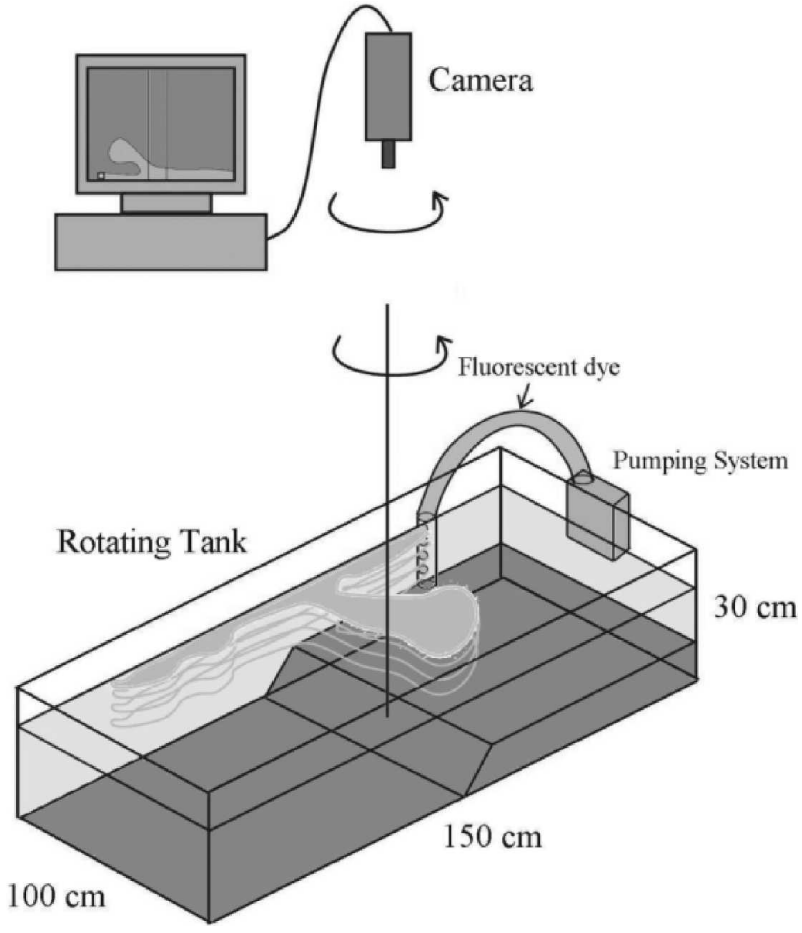

FIG. 1. Schematic view of the experimental setup. The tank rotates in the anticlockwise direction at a constant rate. The flow evolution is recorded by a corotating camera mounted at some distance above the tank.

tube is placed at one of the long sides of the tank and located at a distance $x_{0}$ from the bottom slope (in most cases $x_{0}=25 \mathrm{~cm}$ ). The injected water flows through the small perforations in the tube, arranged at a number of levels over the whole fluid depth and directed parallel to the wall. The end of the tube is closed. Although some disturbances can be created at the outlet of the tube, the current has a nearly uniform vertical distribution. To visualize the current, the injected fluid was colored with fluorescent dye. The evolving dye distributions were recorded with a corotating camera mounted at some distance above the free surface. It is worth mentioning that the injected fluid during an experiment was a small fraction of the total initial volume (about 2\%), and therefore it is assumed that the increase of the water level during the experiment is negligible.

Table 1 shows the experimental parameters of the cases presented here (first four cases), representative of a series of experiments with both left and right geometries (also shown in the table). The Coriolis parameter was varied from 1.5 to $2 \mathrm{~s}^{-1}$, while the current width $\left(L_{0}\right)$ is about $3 \mathrm{~cm}$, and the current speed $\left(U_{0}\right)$ is of order 1-2 $\mathrm{cm} \mathrm{s}^{-1}$. Thus, the Rossby number Ro $=U_{0} /$ $f L_{0}$ of the current is between 0.16 and 0.66 . These mod-
TABLE 1. Experimental parameters. In all cases $W_{0}=4 \mathrm{~cm}$ and $\delta H=6 \mathrm{~cm}$. The sign of the vorticity scale due to the escarpment shown in the fourth column, $f\left(\delta H / H_{0}\right)$, is defined to be negative (positive) for the left- (right)-handed geometry.

\begin{tabular}{|c|c|c|c|c|c|}
\hline Expt & $\begin{array}{c}H_{0} \\
(\mathrm{~cm})\end{array}$ & $\begin{array}{c}f \\
\left(\mathrm{~s}^{-1}\right)\end{array}$ & $\begin{array}{c}f\left(\delta H / H_{0}\right) \\
\left(\mathrm{s}^{-1}\right)\end{array}$ & $\begin{array}{c}x_{0} \\
(\mathrm{~cm})\end{array}$ & Geometry \\
\hline LG1 & 24 & 2.0 & -0.5 & -25 & $\begin{array}{l}\text { Left (shallow } \\
\text { to deep) }\end{array}$ \\
\hline LG2 & 20 & 2.0 & -0.6 & -25 & Left \\
\hline LG3 & 20 & 1.5 & -0.45 & -25 & Left \\
\hline RG1 & 18 & 1.5 & 0.5 & -25 & $\begin{array}{l}\text { Right (deep } \\
\text { to shallow) }\end{array}$ \\
\hline LG4 & 20 & 0.75 & -0.225 & -25 & Left \\
\hline LG5 & 20 & 1.0 & -0.3 & -25 & Left \\
\hline LG6 & 23 & 1.0 & -0.26 & -25 & Left \\
\hline LG7 & 23 & 1.5 & -0.391 & -25 & Left \\
\hline LG8 & 23 & 2.0 & -0.522 & -25 & Left \\
\hline LG9 & 20 & 1.5 & -0.45 & -15 & Left \\
\hline LG10 & 20 & 2.0 & -0.6 & -15 & Left \\
\hline LG11 & 20 & 1.5 & -0.45 & -30 & Left \\
\hline RG2 & 23 & 1.0 & 0.26 & -25 & Right \\
\hline RG3 & 23 & 1.1 & 0.289 & -25 & Right \\
\hline RG4 & 23 & 2.0 & 0.528 & -25 & Right \\
\hline RG5 & 18 & 1.0 & 0.333 & -25 & Right \\
\hline RG6 & 23 & 1.0 & 0.26 & -20 & Right \\
\hline
\end{tabular}

erate values indicate the importance of rotation effects and allow the assumption that shallow water dynamics governs the fluid motion. Furthermore, the experimental results are in very good agreement with QG simulations presented in next sections. It is assumed that the parabolic deformation of the free surface is of secondary importance to the flow evolution. On the other hand, the Ekman period associated with bottom damping, $T_{E} \approx\left(H_{0}^{2} / \Omega \nu\right)^{1 / 2}$ with $\nu$ being the water kinematic viscosity, is about $230 \mathrm{~s}$ (see, e.g., Zavala Sansón and van Heijst 2002). The duration of the experiments is somewhat less than this period, and therefore bottom friction effects are considered unimportant.

\section{b. Coastal current from shallow to deep water}

In all experiments presented here the initial current has the coast to the right. Figure 2 shows the evolution of a coastal flow in the left geometry moving from shallow to deep water (from left to right in the figure). This is experiment LG1 in Table 1, where $f=2 \mathrm{~s}^{-1}$. The current steadily flows down to the slope, where it bifurcates: a small part of it crosses into deep waters, while another portion tends to follow the escarpment $(t=60 \mathrm{~s})$. More important, a dipolar structure is formed from the portion of the current moving off the coast $(t=90 \mathrm{~s})$, and it drifts in the opposite direction of the current, toward the shallow side of the tank. This dipolar vortex is asymmetrical, the cyclonic part being 

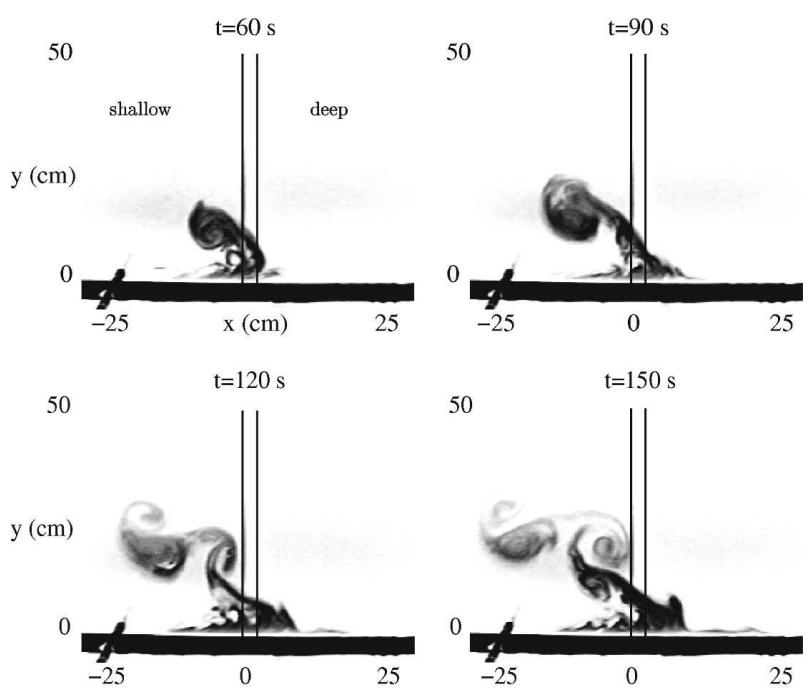

FIG. 2. Top-view photographs showing the evolution of the coastal flow in experiment LG1 (left-handed geometry) during a time span of $150 \mathrm{~s}$ ( $\sim 18$ rotation periods). The current is visualized with dark dye, it is initialized at $x=-25 \mathrm{~cm}$, and it flows from left to right (shallow to deep water). The edges of the slope are marked with vertical lines.

stronger than the anticyclonic side. As a result, the dipole is deflected toward the wall $(t=120 \mathrm{~s})$. Afterward, as the coastal current continues crossing the escarpment, a second dipole is produced with similar initial orientation $(t=150 \mathrm{~s})$.

The bifurcation of the coastal flow and the formation of the dipolar structure were consistently observed in the experiments of Table 1 (most of them were repeated 2-4 times), and these effects can be considered robust results. The essential physical mechanism associated with these processes is the stretching and squeezing of fluid columns as they experience changes in depth. The dipole formation, in fact, is due to the displacement of fluid from the slope and the deep region toward the shallow side of the domain, as the current crosses the slope. This motion implies the squeezing of fluid columns and therefore the formation of a patch of negative relative vorticity, which pairs with the current forming the dipole. It can be noticed, indeed, that the cyclonic part of the dipole is formed with dyed fluid from the current, while the anticyclone is almost undyed.

Another important result is the formation of a weak flow toward the wall, not visible in previous figure. To visualize this inshore flow produced along the slope, Fig. 3 shows a sequence of photographs of the whole domain, where horizontal dye lines were placed perpendicular to the topography before starting the experiment (LG2 in Table 1). The deflection of the dyed
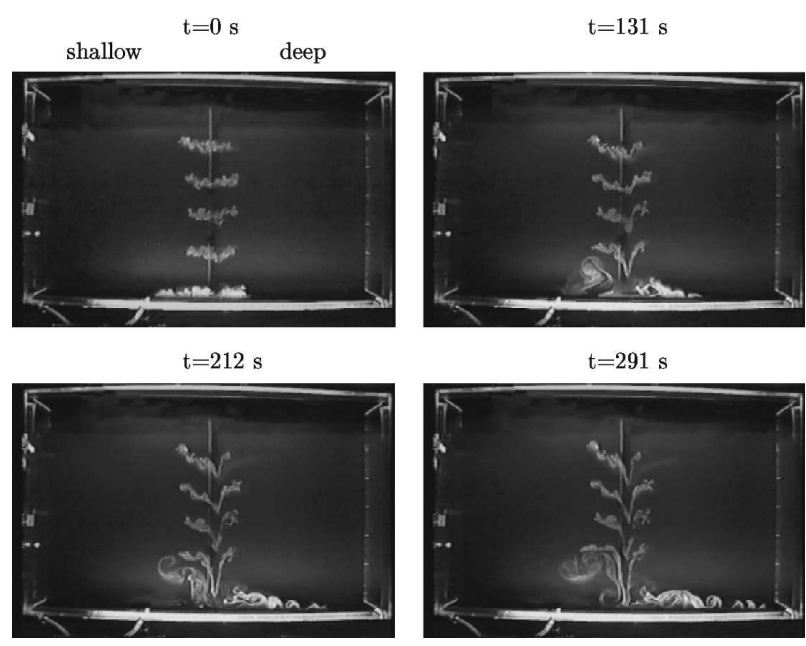

FIG. 3. Top-view photographs of experiment LG2 (left geometry), showing the weak, inshore flow along the topography. The dye lines are nearly perpendicular to the escarpment at the beginning of the experiment.

material lines clearly indicates that an along-slope flow toward the coast is established as the original current bifurcates. This flow is apparently more intense near the coast, eventually reaches the shallow part next to the wall, and contributes to the formation of the dipolar vortex. Since the coastal flow is continuously forced, the water along the slope is also continuously flowing toward the coast, and thus more dipoles can be created.

An additional factor to consider in the experiments is the no-slip boundary condition at the wall. This mechanism induces the formation of relative vorticity at the boundary and contributes to the irregular pattern next to the wall (see also Fig. 2). However, the small-scale structures formed there do not hinder the dipole formation nor the inshore flow along the topography. This will be further shown in next sections by means of numerical simulations using a free-slip condition.

The resultant dipole in each experiment tends to be slightly different. As a result, it can be said that the direction of its trajectory is rather sensitive to small changes in the experimental conditions. Besides, the secondary dipole interacts with the first one, inducing a more erratic behavior. For instance, the dipole formation can take place at a longer distance from the wall, as shown in Fig. 4, which presents an experiment with lower water depth (LG3 in Table 1). In this case, the bifurcated current along the slope seems to travel farther off the coast before the dipole is formed. The difference with the experiment shown in Fig. 2 can be appreciated at $t=90 \mathrm{~s}$ : in that case the dipole is following a diagonal trajectory, while in the second situation the dipole moves perpendicular to the topography. 

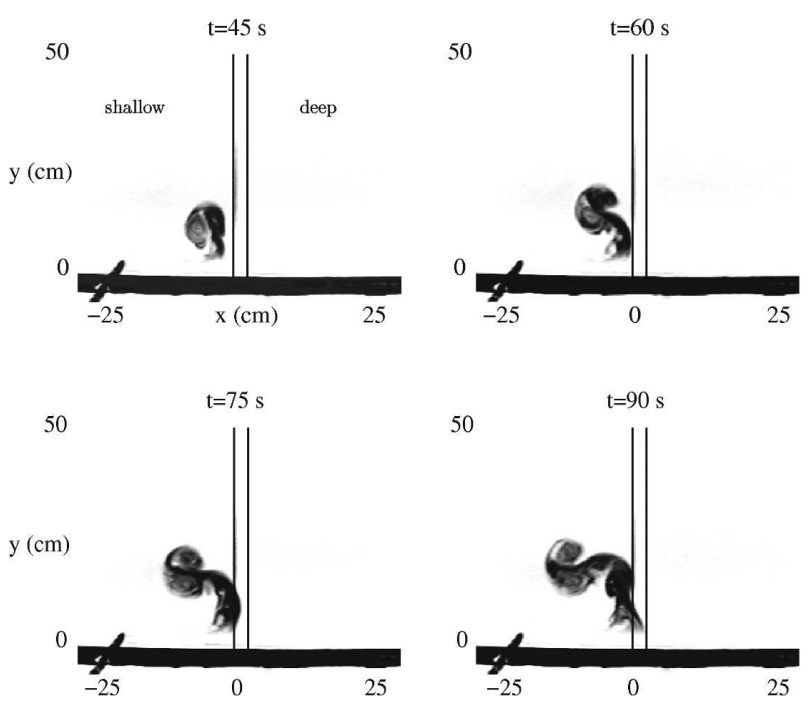

FIG. 4. Top-view photographs of experiment LG3 (left geometry). The dipole formation takes place farther off the coast, relative to the experiment in Fig. 2.

\section{c. Coastal current from deep to shallow water}

Figure 5 shows a sequence of photographs of a current flowing from deep to shallow water, again with the coastal wall to the right. In this case, the current is produced at the upper-right part of the photographs and moves leftward. The outlet is at $25 \mathrm{~cm}$ from the slope and the current is produced in a similar fashion as in previous experiments. The experiment is denoted RG1 in Table 1. When the current reaches the slope,
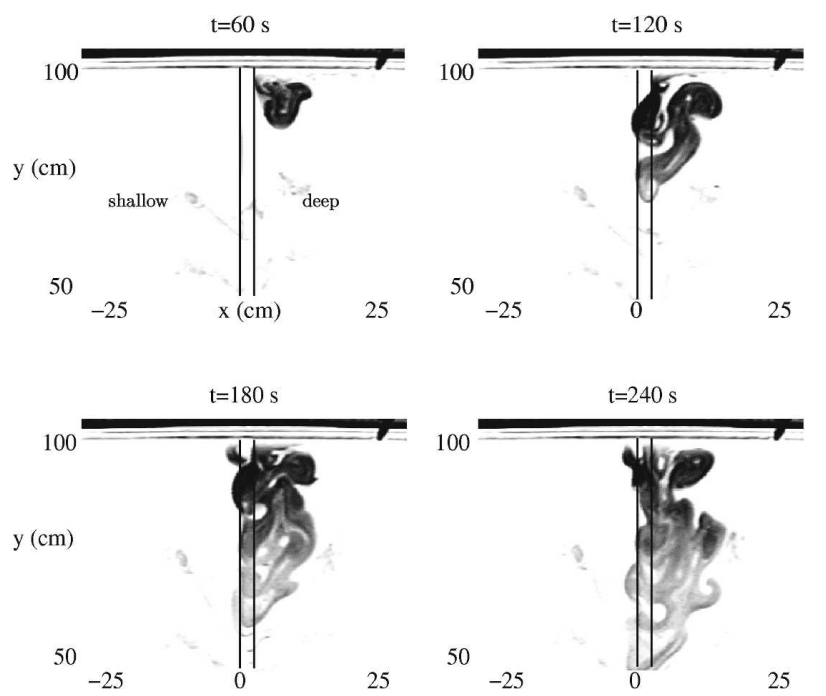

FIG. 5. Top-view photographs of experiment RG1 (right-handed geometry). The current flows from right to left (deep to shallow water) and is deflected along the topography. This process continuously occurs despite the irregular behavior of the current. the flow is almost completely deviated and moves along the topography, perpendicular to the coast $(t=60-120$ s). Thus, a quasi-permanent current is established with a $90^{\circ}$ deflection, which persists during the rest of the experiment $(t=180-240 \mathrm{~s})$.

This behavior is predicted by the analytical results from C99, based on quasigeostrophic dynamics and smooth coastal currents. One of the most remarkable results from these types of experiments is that the current deflection continuously takes place despite the complex structure of the flow. As shown in the figure, the dye distributions show a complicated turbulent flow, which even so follows the topography contours. In addition, such a behavior remains for many rotating periods of the system (almost 30). It is concluded therefore that the current deflection in the presence of a perpendicular topographic feature (like the slope of the present experiment) is a characteristic behavior of a coastal current regardless of its detailed structure.

The irregular character of the current may arise from several factors, such as the no-slip boundary condition at the wall, which continuously induces the creation of relative vorticity. This is manifested in the dye patterns at the intersection of the wall and the topography (see $t=60-120 \mathrm{~s}$ ). Once the current is flowing along the slope, it maintains its irregular behavior.

\section{Quasigeostrophic numerical simulations}

In this section, numerical simulations of a QG current along a vertical wall in the presence of a topographic slope are presented. Essentially, the model solves the QG vorticity equation by means of a finite differences scheme (Orlandi 1990; Serravall 1999):

$$
\frac{\partial \omega}{\partial t}+J(\psi, q)=A \nabla^{2} \omega
$$

where $\omega=\partial v / \partial x-\partial u / \partial y$ is the relative vorticity, with $\mathbf{u}=(u, v)$ being the horizontal velocity components in the $x$ and $y$ directions, $A$ is the eddy viscosity coefficient (which is set to be small as possible consistent with numerical stability), $\psi$ is a streamfunction such that $\omega=$ $\nabla^{2} \psi$, and $q$ is the potential vorticity defined as

$$
q=\omega-f \frac{\Delta H}{H} .
$$

Here, $f$ is the constant Coriolis parameter, $\Delta H(x, y)$ are small topographic variations, and $H$ is a reference depth such that $|\Delta H| / H \ll 1$ all over the domain.

To nondimensionalize the evolution equation, consider a coastal current of width $L$ and velocity $U$, im- 
posed next to a straight wall. The corresponding scale for the streamfunction is $L U$, for the vorticity is $U / L$, and time scales as $L / U$. Using these parameters the vorticity equation yields

$$
\frac{\partial \omega^{*}}{\partial t^{*}}+J\left(\psi^{*}, q^{*}\right)=\frac{1}{\operatorname{Re}} \nabla^{2} \omega^{*},
$$

where $\operatorname{Re}=U L / A$ is the Reynolds number and asterisks indicate nondimensional variables. The nondimensional potential vorticity is defined as

$$
q^{*}=\omega^{*}+h^{*},
$$

where the topographic term is

$$
h^{*}(x, y)=-f \frac{\Delta H(x, y)}{H} \frac{L}{U} .
$$

For the cases studied here there are only topographic variations in the $x$ direction. Consider that the current flows from a region with depth $H$ toward a region with depth $H+\Delta H(x)$, where $\Delta H(x) \geq 0$ in the left-handed geometry (i.e., when flowing from shallow to deep water) and $\Delta H(x) \leq 0$ in the right-handed geometry (from deep to shallow water). The total increment of the topographic term $h^{*}$ is defined as

$$
\delta h^{*}=-f \frac{\delta H}{H} \frac{L}{U},
$$

where $\delta H$ is the total depth increment: $\delta H=\max \Delta H>$ 0 in the left geometry, and $\delta H=\min \Delta H<0$ in the right geometry. Thus, the magnitude of $\delta h^{*}$ indicates the importance of the topographic effects due to the escarpment, and its sign defines the geometry $\left(\delta h^{*}<0=\right.$ left; $\delta h^{*}>0=$ right).

Using a Cartesian reference system, the coastline is defined as the $x$ axis and the perpendicular direction, along the topography, is defined as the $y$ axis. The vorticity equation is discretized over a nonuniform grid with $193 \times 129$ points, where the density of grid points is higher next to the topographic slope. The nondimensional size of the numerical domain is a $20 \times 10$ rectangle. Looking toward the positive $x$ direction, the slope starts at $x=9$, and has a width $W^{*}$, which varies from 0.5 to 4 for several runs. To emphasize that the essential physics here is inviscid, we have neglected bottom drag effects and maintain a finite Reynolds number only to ensure numerical stability. In all simulations, the Reynolds number is set at $\mathrm{Re}=3000$. The boundary condition used at the coastline is free-slip, while a radiation condition (Orlanski 1976) is applied for the open boundaries. A variable time step adjusted by the Courant number criterion was used.
TABLE 2. Numerical parameters of simulations shown in Figs. 6 and $9-12$.

\begin{tabular}{crll}
\hline \hline Simulation & $\delta h^{*}$ & $W^{*}$ & \multicolumn{1}{c}{ Geometry } \\
\hline S1 & -0.5 & 0.5 & Left (shallow to deep) \\
S2 & -0.5 & 2.0 & Left \\
S3 & -4.0 & 0.5 & Left \\
S4 & 2.0 & 0.5 & Right (deep to shallow) \\
S5 & 1.0 & 0.5 & Right \\
\hline
\end{tabular}

The dimensional initial condition is a current profile given by

$$
u(y)=U \exp (-y / L)
$$

which is prescribed far enough from the escarpment in order to minimize the effect of a finite geometry. The nondimensional form of the profile is $u^{*}\left(y^{*}\right)=$ $\exp \left(-y^{*}\right)$. Note that this current has a maximum value at the wall, and it has positive relative vorticity for all $y^{*}$.

The numerical experiments consist of several runs (see Table 2) varying the topographic term $\delta h^{*}$, which measures the importance of changes in depth of fluid columns due to the topography $(f \delta H / H)$, as compared with the vorticity associated with the current $(U / L)$. Another important parameter to be varied is the nondimensional width of the slope $W^{*}$.

\section{a. Shallow to deep flow}

The first case to analyze is a coastal current flowing from shallow to deep water, as shown in Fig. 6. The fluid motion takes place along the $x$ direction from left to right. Here, $\delta h^{*}=-0.5$ and $W^{*}=0.5$, which can be interpreted as a small topographic step and a narrow slope, respectively. At times $t=10-20$ the current approaches to the topographic slope. It is important to notice the formation of positive relative vorticity over the slope and next to the wall (and farther downstream), due to stretching effects as fluid pushed by the current crosses the topography. Indeed, by considering potential vorticity conservation, fluid columns are vertically stretched and positive relative vorticity is generated. At a longer distance from the wall, negative relative vorticity is also created due to squeezing effects. This anticyclonic patch can also be considered as part of the topographic radiation over the slope, as shall be discussed below. At $t=30-40$ the coastal current head has crossed the topography, while the negative vorticity over the slope pairs with the current, forming a dipolar structure at $t=50-60$. This process occurs in a similar fashion as in the laboratory experiments. Afterward, a secondary dipole starts to form at $t=70-80$.

Once formed, the dipole drifts toward the shallow 


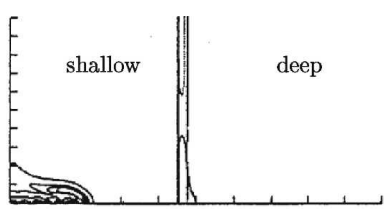

$t=10)$

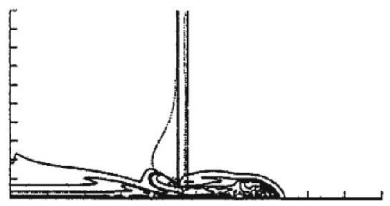

$t=30$ )

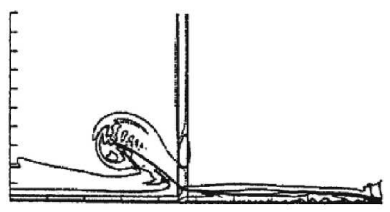

$t=50$

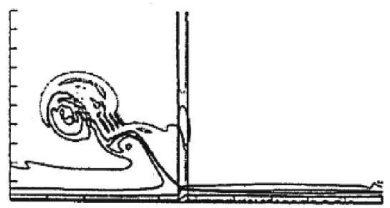

$t=70)$


$t=40)$

$t=60)$

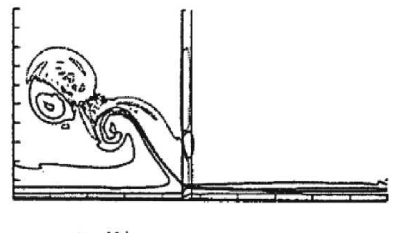

$\left.t=B_{0}\right)$



FIG. 6. Vorticity contours calculated from simulation $\mathrm{S} 1$ in the left geometry $\left(\delta h^{*}=-0.5, W^{*}=0.5\right)$. This figure illustrates the dipole formation and its subsequent drift into the shallow region. Solid (dotted) contours correspond to positive (negative) relative vorticity. The contour interval is $\Delta \omega^{*}=0.5$.

region and moves away from the coast. This motion is maintained approximately with a straight trajectory during the time span of the simulation. Figure 7 shows the trajectories of the vorticity maximum and minimum after the dipole formation, which are oriented at an

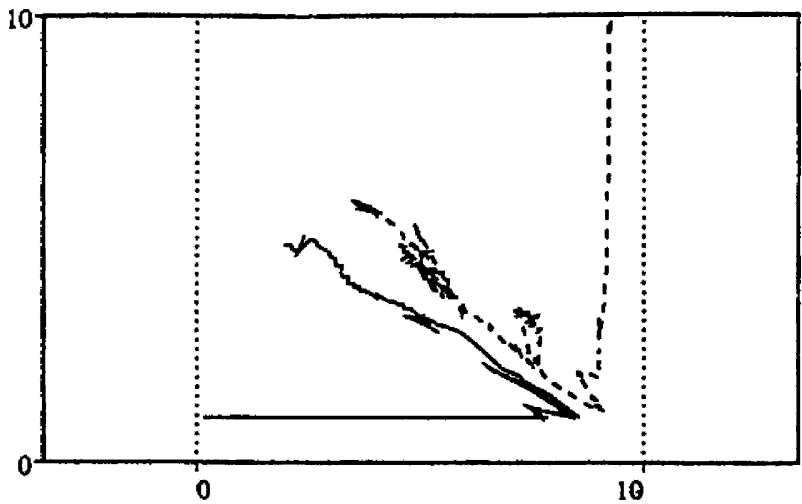

FIG. 7. Trajectories of the vorticity extrema of the dipolar structure formed during the current-slope interaction in simulation S1. angle of approximately $38^{\circ}$ with respect to the negative $x$ direction. How does this direction depend on $\delta h^{*}$ and $W^{*}$ ? To answer this question, Fig. 8 presents the measured direction of the dipole trajectory as a function of $\delta h^{*}$. The angles were measured at a time when the two opposite signed vortices come close enough together to form a compact coherent dipole. The angle is that made by the line tangent to the zero vorticity contour in the middle of the dipole at that time in each simulation. Given the possible variation in defining the best time to make the measurement and some error in defining the tangent to the zero vorticity contour, it is estimated that a reasonable error bar for the measurements is $3^{\circ}$. The important point here is that, even with this error margin, the trend is well defined: in general, it is found that the dipole trajectory is strongly affected by changes in $\delta h^{*}$, while additional simulations showed that it is not altered by changes in the slope width $W^{*}$. This behavior can be better understood by considering the effect of an increase of the topographic height $\delta h^{*}$. This causes greater squeezing of fluid columns over the slope, inducing an intensification of the negative vorticity patch over the topography. As a consequence, the dipolar structure will tend to drift away from the coast, because of the influence of the stronger negative part of the dipole. In contrast, changing the values of $W^{*}$ produced little effect.

The relative vorticity of alternating sign produced over the topography deserves an additional comment. It may be considered a manifestation of topographic waves over the slope, which tend to drift along topography contours, with shallow water to the right. An interpretation of this is to consider that the topographic



FIG. 8. Orientation angle $\alpha\left({ }^{\circ}\right)$ of the dipole trajectory with respect to the negative $x$ axis, measured in numerical simulations for different $\delta h^{*}$ 
waves have a phase velocity toward the local "westward" direction. In general, the topographic "north" is pointing upslope, and therefore the "west" corresponds with the negative $y$ axis in the present case (see e.g., Carnevale et al. 1991). The topographic radiation can be observed in Fig. 9, where the case of a wider slope, $W^{*}=2$, is presented. Note that a row of patches with alternating vorticity is generated, which tend to drift toward the wall. For instance, at $t=50$ a negative vorticity patch next to the wall has already paired with the current and, farther away from the coast, a cyclonic and an anticyclonic vorticity area have been formed. At $t=$ 60 these structures already drifted toward the coast.

The flow behavior when $\delta h^{*}$ is increased is shown in Fig. 10, where a case with $\delta h^{*}=-4$ and $W^{*}=0.5$ is presented. First, notice that the positive vorticity created next to the wall, in the deep part (see $t=10-20$ ), is more intense than in the case with $\delta h^{*}=-0.5$ (Fig. 6). This result is to be expected since the increased depth difference enhances stretching effects when fluid columns experience changes in depth. It was found in Fig. 8 that the angle of the dipole direction is more pronounced when $\delta h^{*}$ is increased, thus tending to follow the topographic contours. This is seen at $t=30-40$,

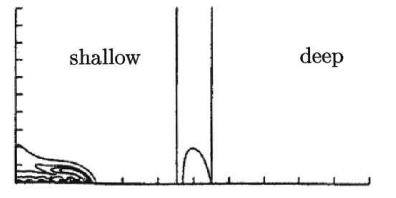

$t=10$ )

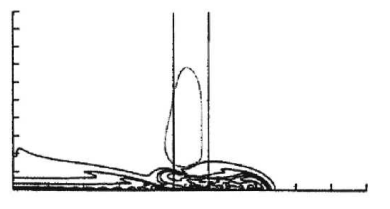

$t=30$ )

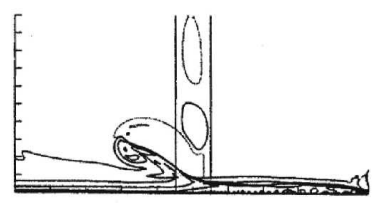

$t=50$ )

$t=70$



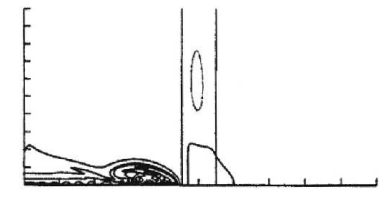

$t=20$ )

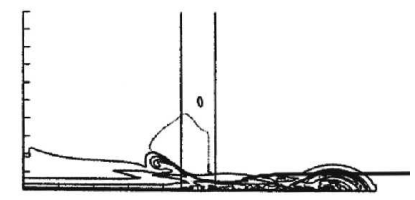

$t=40$ )

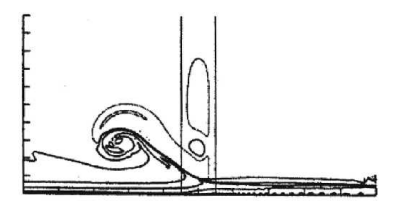

$t=60$ )

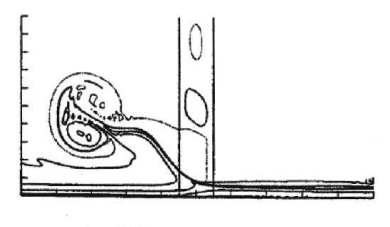

$t=80$ )
FIG. 9. Vorticity contours calculated from simulation S2 in the left geometry $\left(\delta h^{*}=-0.5, W^{*}=2\right)$. The alternating vorticity cells at the slope indicate the occurrence of topographic waves.

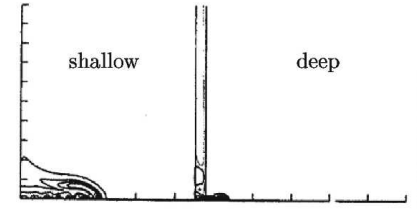

$t=10)$

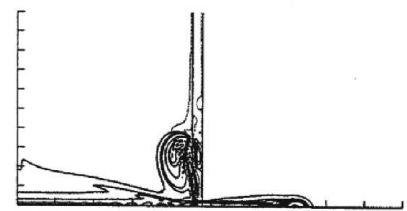

$t=30$ )

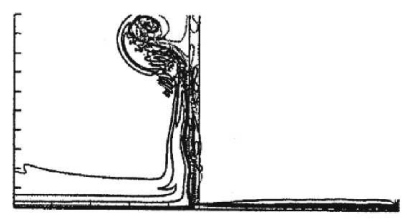

$t=50$ )

FIG. 10. Vorticity contours calculated from simulation S3 in the left geometry $\left(\delta h^{*}=-4, W^{*}=0.5\right)$. For increasing $\delta h^{*}$ the flow is deflected along the topography, and the dipole is formed far from the wall.

where the flow bifurcates, part of it following the coastline, and the other part moving along the slope. Eventually, the dipolar vortex is formed, which detaches from the escarpment at $t=50$. Since this process continues, a second, and even a third dipole, can be also formed (not shown here).

\section{b. Deep to shallow flow}

The dynamics of the coastal current when flowing from deep to shallow water (with the coast to the right) is completely different from that described above, as shown in the laboratory experiments. In this case, the current might be completely deflected from the coastline with offshore motion along the topography. Figure 11 shows a sequence of vorticity contours of a coastal flow from deep to shallow water (note that the deep region is at the left side of the domain, in contrast with previous figures). The flow parameters are $\delta h^{*}=2$ and $W^{*}=0.5$. The current is completely deflected from the coast and moves along the topography contours, in a similar fashion as in the experiment shown in Fig. 5. When the current reaches the slope, negative vorticity is produced at the shallow side, due to squeezing effects $(t=30-40)$. The jet formed clearly has a positive vorticity flank at the deep (left) side. After some time, the nose of the current has left the domain, and a nearly uniform flow has been established. This is in relative contrast with the laboratory experiment shown in Fig. 


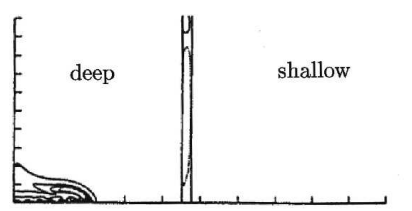

$t=10)$

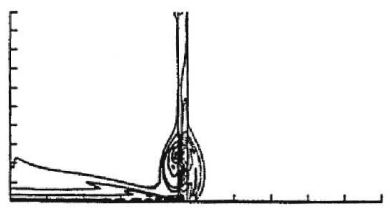

$t=30)$

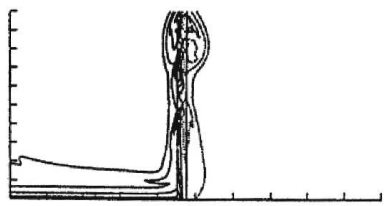

$t=50)$

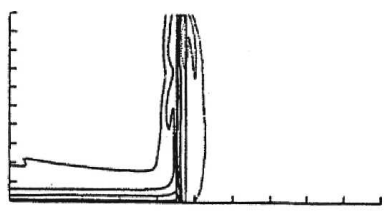

$t=70)$

FIG. 11. Vorticity contours calculated from simulation S4 in the right geometry $\left(\delta h^{*}=2, W^{*}=0.5\right)$. The current is deviated and flows along the escarpment.

5 , where the current is quite irregular and turbulent. It is important to note here, however, that the flow along the topography is a very robust result both experimentally and numerically. In addition, this behavior was also analytically described by $\mathrm{C} 99$ for coastal currents in a right-handed geometry. Another important feature of the observed flow behavior (which will be further discussed in the last section) is that dipole formation is completely absent in this case, other than the dipolar structure that leads the current along the topography.

Of course, the deflection of the current strongly depends on $\delta h^{*}$. For instance, a vanishing change of depth $\delta H\left(\delta h^{*}\right.$ small or null) implies that the current simply moves along the coast with no deflection and is only subject to its inherent dynamics. Figure 12 shows the case with $\delta h^{*}=1$ and $W^{*}=0.5$. Initially $(t=10-20)$, the flow behavior is identical as in previous case. For later times, however, the current is bifurcated when encountering the slope. Although some complicated vortex activity is observed at the topography next to the wall, part of the current crosses to the shallow side, and another part moves offshore. The structure of the along-slope flow is weaker and less uniform, when com-
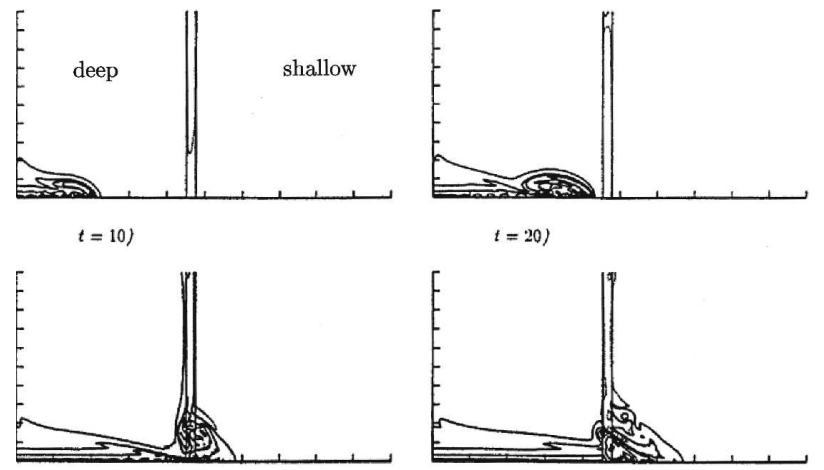

$t=30$ )

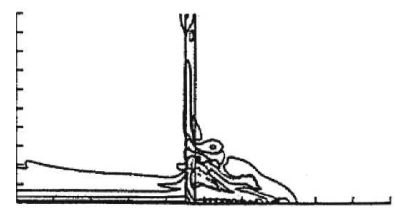

$t=30)$
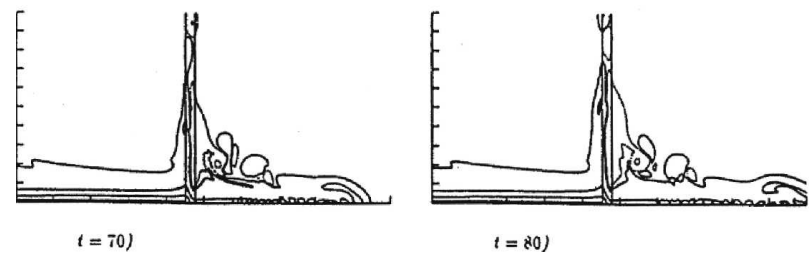

FIG. 12. Vorticity contours calculated from simulation S5 in the right geometry $\left(\delta h^{*}=1, W^{*}=0.5\right)$. For smaller $\delta h^{*}$ the current is bifurcated: part of it follows the coastline, while the other part follows the topography.

pared with previous figures. This result is easily interpreted by considering that a lower escarpment (e.g., lower $\delta h^{*}$ ) reduces the production of negative relative vorticity at the shallow side, which in turn is responsible for the current deviation. As a result, only part of the current is deflected.

\section{Analysis with passive tracers}

One of the most important, and complicated, processes observed in the laboratory experiments and the numerical simulations is the dipole formation for the left geometry and the corresponding inshore current along the topography. These are due to the fluid exchange between the shallow and the deep basins, derived from the nonlinear character of the flow and the geometry itself. This behavior can be further analyzed by means of numerical simulations in which passive tracers are initially placed at the shallow or at the deep side, or at the slope. The simulations discussed in this section are performed with a finite differences code similar to the used in previous section. The original scheme was initially developed by Verzicco and Or- 
landi, and later modified by Zavala Sansón in order to include variable topography with either the shallow water or the QG formulation (Zavala Sansón and van Heijst 2002).

\section{a. Fluid exchange between basins}

In this section a numerical simulation in the left geometry showing the communication between the shallow and deep regions is presented. In this case, the flow parameters are adjusted to the laboratory values in order to show the QG character of the experiments. The domain is a $150 \mathrm{~cm} \times 100 \mathrm{~cm}$ rectangle, and the depth field is prescribed as in (1). The corresponding depth at the shallow and deep parts of the domain are 18 and 24 $\mathrm{cm}$, respectively, the width of the slope is $5 \mathrm{~cm}$, and the Coriolis parameter is $f=1.5 \mathrm{~s}^{-1}$. Considering an initial velocity profile as in (8), the current parameters are set as $L=3 \mathrm{~cm}$, and $U=3 \mathrm{~cm} \mathrm{~s}^{-1}$. This initial profile is prescribed at $x<-25 \mathrm{~cm}$ in order to simulate the production of the current at that position.

To compare a QG simulation with a typical laboratory experiment, a linear bottom friction term is added in the dimensional vorticity Eq. (2):

$$
\frac{\partial \omega}{\partial t}+J(\psi, q)=\nu \nabla^{2} \omega-\frac{1}{2} E^{1 / 2} f \omega,
$$

where $E=2 \nu / f H^{2}$ is the Ekman number. The kinematic viscosity of water is $\nu=0.01 \mathrm{~cm}^{2} \mathrm{~s}^{-1}$, and the mean depth is $H=21 \mathrm{~cm}$. In addition, a no-slip boundary condition is imposed at the coast.

Figure 13 presents the evolution of three sets of passive tracers initially placed at the shallow part, on the slope, and at the deep side of the domain (see $t=0 \mathrm{~s}$ ). Note that there are no tracers in the region between the black particles (initially in the current) and the slope. This situation is similar to the laboratory experiments, where initially the fluid between the outlet of the tube and the slope is undyed.

At $t=30 \mathrm{~s}$, the black particles arrive next to the slope, displacing the darker and "white" tracers (in front of the current) over the slope, toward the deep region. More important, farther offshore there is entrainment of fluid from the slope (darker tracers) toward the shallow part of the domain. Consequently, particles from the deep side (gray) start to move onto the slope. At $t=60-90 \mathrm{~s}$, the dipole formation occurs: the cyclonic part is essentially formed by fluid originally in the current, while the anticyclonic side is formed by particles initially on the slope and others from the deep side. The dipole moves onto the shallow region of the domain, eventually turning with a leftward inclination $(t=120-150 \mathrm{~s})$. This is due to its asymmetrical struc-
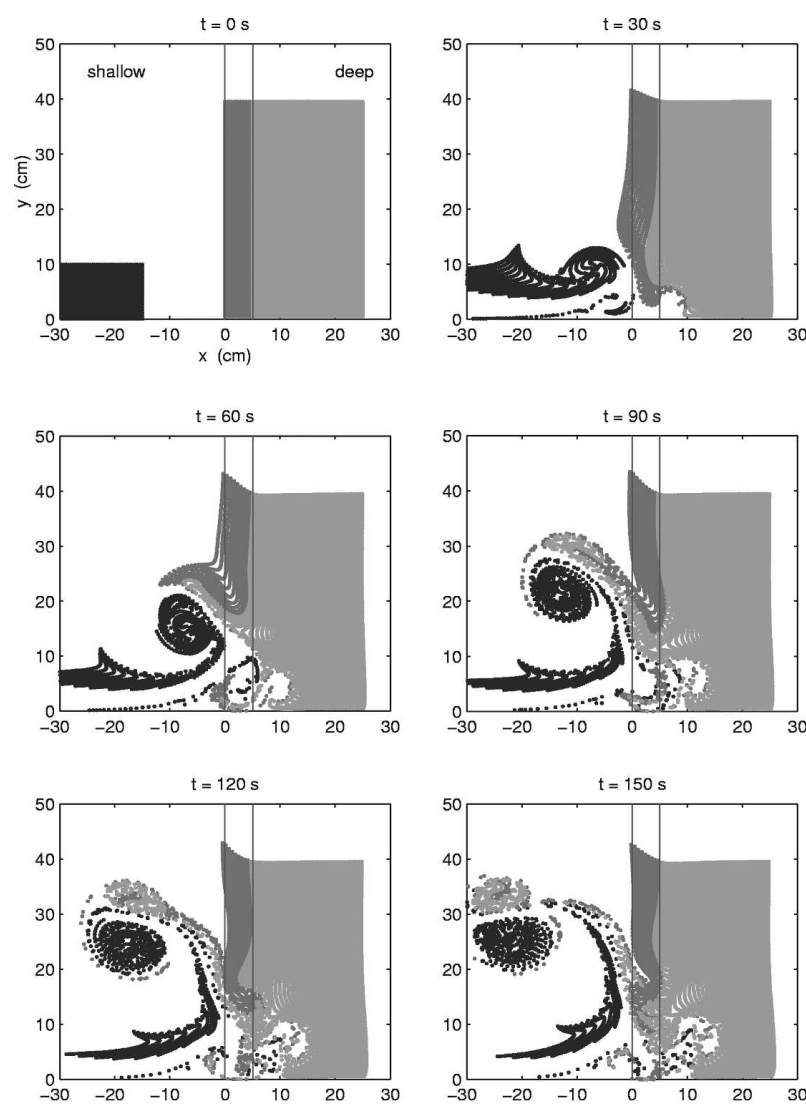

FIG. 13. Evolution of passive tracers calculated from a simulation in the left geometry using typical experimental parameters. Initially, three patches of particles are arranged in rectangular areas: 1) 1000 black particles at the shallow region inside a $20 \mathrm{~cm}$ $\times 10 \mathrm{~cm}$ rectangle, 2) 1000 particles at the slope inside a $5 \mathrm{~cm} \times$ $40 \mathrm{~cm}$ area, and 3) 4000 gray particles at the deep region inside a $20 \mathrm{~cm} \times 40 \mathrm{~cm}$ rectangle. A no-slip boundary condition is used at the wall, and linear bottom friction is included.

ture: the positive part (formed by the current) is stronger than the negative side (formed by squeezing effects as fluid flows into the shallow region). This is the mechanism described in the experimental section. In contrast to the experiments, however, the simulation is not continually forced; as a result, a secondary dipole is only weakly formed at subsequent times (not shown here).

The flow analysis with tracers shows that the laboratory experiments are well represented, at least qualitatively, by the QG dynamics. Note also that close to the wall a complicated pattern is formed. This is essentially a result of the topographic waves trying to move with shallow water on its right and then being obstructed by the wall. Besides, the no-slip boundary condition imposed to simulate the laboratory experiment implies the formation of negative relative vorticity at the wall before the current reaches the slope. For clarity, Fig. 14 

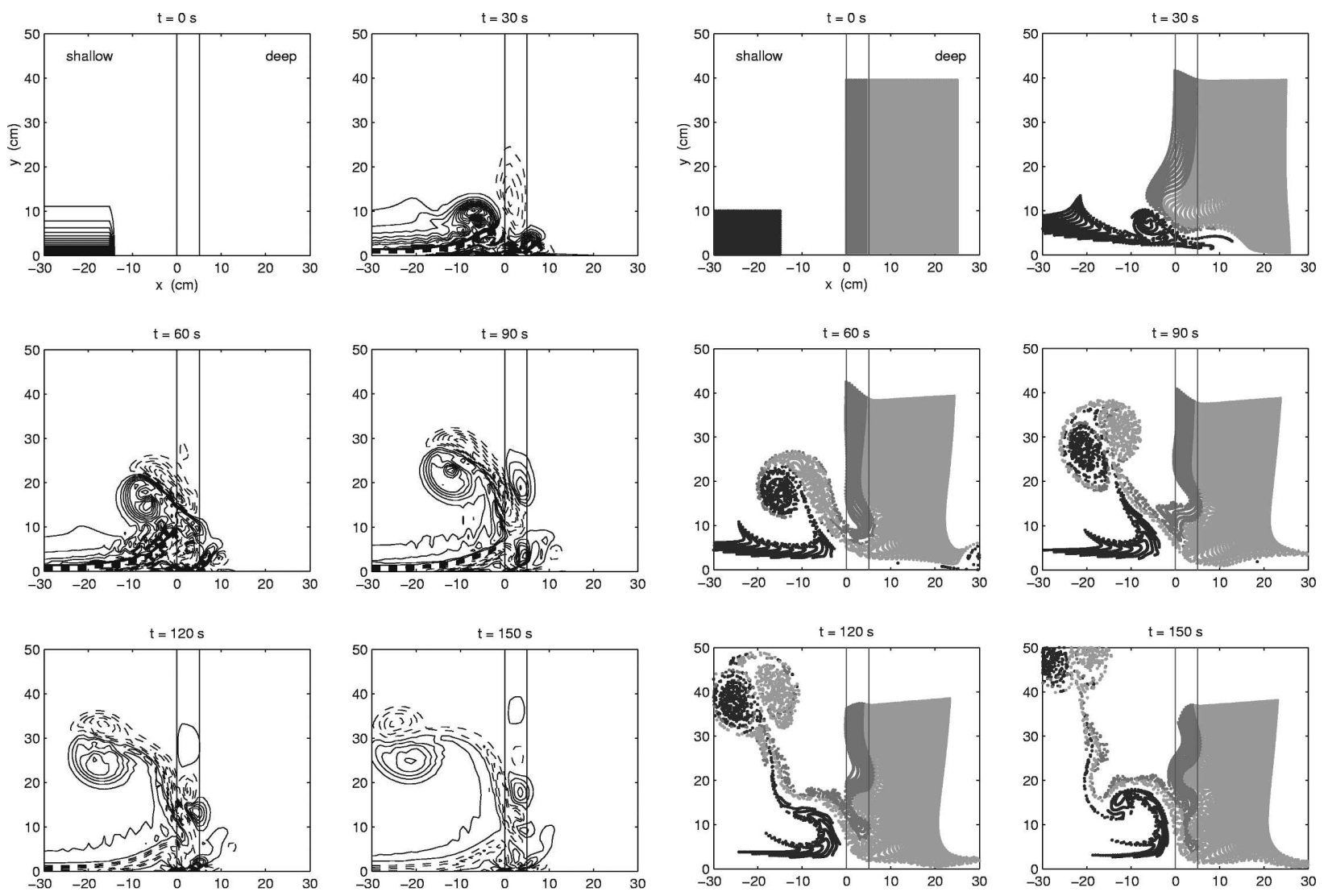

FIG. 14. Vorticity contours calculated from the simulation shown in Fig. 13. The contour interval is $0.05 \mathrm{~s}^{-1}$, and the maximum (minimum) vorticity is $1(-1) \mathrm{s}^{-1}$. Solid (dashed) contours correspond to positive (negative) relative vorticity.

presents the corresponding vorticity contours at the same times as in the previous figure. The vorticity created at the wall contributes to the formation of the complicated flow pattern next to the step. Such a mechanism, however, does not hinder the dipole formation, which is an inviscid process, as shown in the numerical simulations of previous sections, where a free-slip condition was used. However, the free-slip condition "smooths" the complicated pattern next to the wall and, therefore, it will be used in the next section to analyze the flow over the slope.

\section{b. Influence of the coastal boundary condition}

The dipole formation is a mechanism due only to squeezing effects; therefore, it does not depend on the coastal boundary condition or on the bottom friction. This was shown in the QG simulations of section 3, where a free-slip boundary was used. Here, Figs. 15 and 16 present the corresponding tracers and vorticity contours calculated from an additional numerical simula-
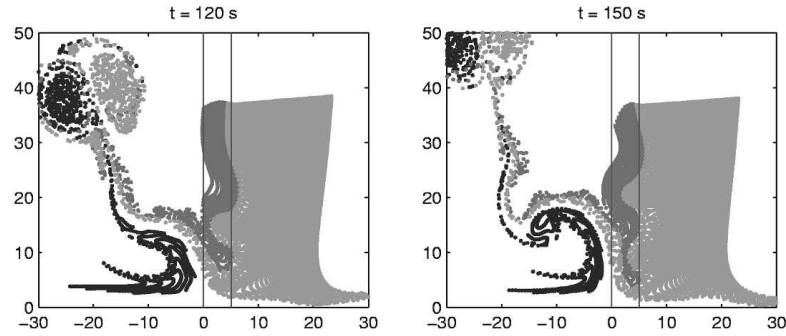

FIG. 15. Evolution of the same arrangement of passive tracers shown in Fig. 13, but now using a free-slip boundary condition at the wall, and with no bottom friction.

tion with a free-slip condition at the wall and with no bottom friction. The results are shown for the same times as in Figs. 13 and 14 so that they can be directly compared.

In contrast with the no-slip case, the "white" fluid in front of the current slips along the wall, crossing the slope and moving farther to the right, deep part of the domain $(t=30 \mathrm{~s})$. Even though, there is exchange of fluid between the two basins, leading to the formation of the dipole in the shallow region. This structure is formed approximately at the same time as in the no-slip case $(t=60-90 \mathrm{~s})$. Note, however, that the dipole in the present case is almost symmetrical and therefore it moves offshore approximately in a straight line. It is evident at $t=60 \mathrm{~s}$ that the amount of fluid crossing the topography from deep to shallow water (gray tracers) is greater than in the no-slip case. This implies a stronger anticyclonic part of the dipole and, therefore, a more symmetrical structure. Such a difference reaffirms that the physical mechanism involved in the dipole formation and detachment are squeezing effects associated with the communication between the regions. 

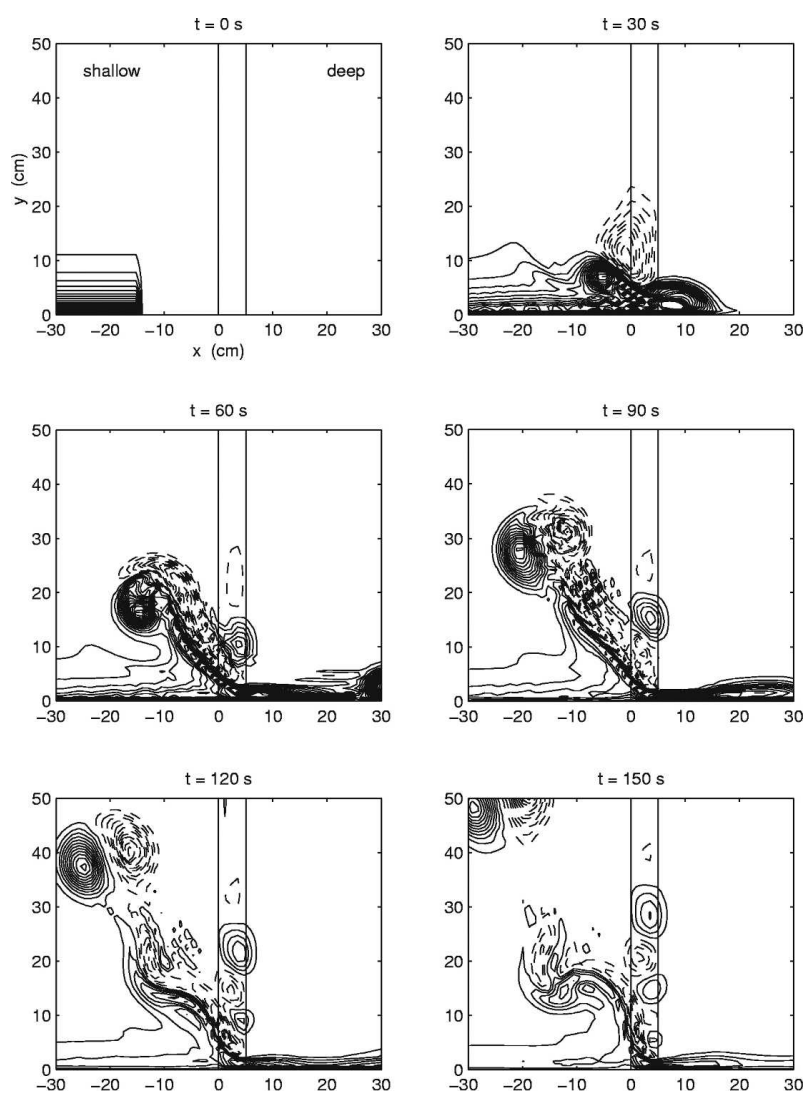

FIG. 16. Vorticity contours calculated from the simulation shown in Fig. 15. Contours are as in Fig. 14.

The motion of the set of particles over the slope in Fig. 15 is of particular interest. Note that the arrangement of tracers acquires an undulatory shape due to the topographic radiation over the slope, which was described before. This motion is clear from the vorticity contours shown in Fig. 16, especially at $t=120-150 \mathrm{~s}$ when alternate patches of opposite-signed vorticity are formed. It must be recalled that the phase velocity of the topographic waves is directed toward the coast, which is the corresponding "west" direction.

Furthermore, it can be shown that the tracers slightly move toward the wall, establishing the inshore current described before. Figure 17 shows the same simulation but now using two different sets of particles: one in the current, as in previous cases, and 10 lines of tracers perpendicular to the escarpment, as in the experiment shown in Fig. $3(t=0 \mathrm{~s})$. A number of dashed, horizontal lines have been drawn in order to visualize the weak displacement of particles along the slope. Clearly, the passive lines close to the wall are advected inshore, especially at the deep part of the escarpment. This motion, however, seems weaker than in the experiments, which is most likely due to the lack of forcing in the simulation (in contrast with the experiments).


FIG. 17. Evolution of two groups of passive tracers in the same numerical simulation shown in Fig. 15: 1) 1000 particles at the shallow region inside a $20 \mathrm{~cm} \times 10 \mathrm{~cm}$ rectangle and 2) 1000 particles at the slope, arranged in 10 lines perpendicular to the topography. Dashed, horizontal lines are drawn in order to show the along-slope displacement of the particles.

\section{Discussion and conclusions}

The evolution of a coastal current encountering a topographic slope perpendicular to the coast has been studied by means of laboratory experiments and numerical simulations. Two cases have been analyzed: (i) the left-handed geometry with the coastal current flowing from shallow to deep water and (ii) the righthanded geometry with the current flowing from deep to shallow water. Since the experimental boundary currents were created by a source method (inducing a sur- 
face elevation), they always flow with the coast on their right.

In the left geometry, a complicated flow pattern evolves at the coast when the current approaches the topography. In linear theory this area corresponds with the singularity associated with the current when trying to move with shallow fluid on the right, and is obstructed by the presence of the coast. There is flow across the escarpment: from shallow to deep water next to the wall, and from deep to shallow water farther offshore. The communication between the two basins leads to the formation of a dipolar structure, which moves back into the shallow region. The cyclonic part of the dipole is formed by the positive relative vorticity associated with the coastal current. The anticyclonic part is formed by fluid crossing the slope from the deep side of the domain, therefore acquiring negative relative vorticity due to squeezing effects. This process is clearly visible in Fig. 13, where the positions of passive tracers at different regions were tracked. The direction of the dipolar structure depends on the flow parameters (section 3) and also on the boundary condition at the coast (section 4). A similar mechanism has been reported before for vortices approaching a steplike topography, where cyclones cannot cross a step-down escarpment and they are "reflected" toward the shallow region (Zavala Sansón et al. 1999).

It is evident that the flow evolution depends on the change of the depth due to the topography. This behavior can be represented by the nondimensional number given in (7), which can be interpreted as the ratio of the vorticity scale associated with the escarpment $(f \delta H /$ $H)$ and the vorticity scale of the coastal current $(U / L)$. This number can be rewritten as $\delta h^{*} \sim(\delta H / H) / \varepsilon$ with $\varepsilon=U / f L$ the Rossby number. When the step height is further increased, it was found that the dipole tends to move perpendicular to the coast, along the step, as shown in Fig. 8 and in the numerical simulation in Fig. 10. From these results, it can be said that for very large steps the coastal current is deflected in order to follow the topography. This assertion is consistent with the results of Spitz and Nof (1991) who reported experiments in a similar geometry but using a very large escarpment. In their experiments with the left geometry the flow parameters are $\delta H=18, H=21 \mathrm{~cm}$ and $\varepsilon \sim$ 0.16 , so $\delta h^{*} \approx 5.4$. As a result of this large value, their observed current flows along the step reaching the opposite side of the tank without the formation of a dipolar structure. In contrast, Stern and Austin (1995) reported experiments with a lower $\delta h^{*}(\approx 1.75)$, and thus the dipole is formed near the wall.

Another important feature of the left-handed geometry is the formation of an inshore, weak flow along the topography (see Fig. 3), which moves with shallow water on its right. This flow can be understood from the Taylor-Proudman theorem, which states that for slow motions (low Rossby number) vertical motions are suppressed, and therefore fluid columns tend to conserve their depth. Such a behavior, however, does not occur near the coast, where the nonlinear character of the flow allows the communication between the basins. There is another simple theoretical explanation for the generation and direction of topographic currents based on statistical theory. Conservation of energy and enstrophy starting from a random initial condition will predict the maximum entropy or maximum probability state with a current along the topography in the direction of the topographic Rossby wave phase velocity (see, e.g., Salmon et al. 1976; Carnevale and Frederiksen 1987). Furthermore, topographic waves were observed in the numerical simulations. The alternating cells of positive and negative vorticity over the slope are the manifestation of such waves traveling "westward," that is, toward the wall.

On the other hand, the right geometry shows a very different behavior. In this case, the current is deflected along the topography and moves offshore with shallow water on its right, as theoretically predicted by C99. A remarkable result is that the current deflection continuously occurs despite the complex structure of the flow. This indicates that the detachment from the coast to follow the topographic contours is a characteristic behavior of a coastal current in the right geometry. Such a behavior can also be explained by the fact that in this geometry topographic waves travel in the same direction as the bifurcated current, in contrast with the left geometry.

Acknowledgments. One of the authors (GFC) is grateful for financial support from National Science Foundation through Grant OCE 01-29301 and the Ministero Istruzione Universita e Ricerca (MIUR D.M. 26.01.01 n. 13).

\section{REFERENCES}

Allen, S. E., 1988: Rossby adjustment over a slope. Ph.D. thesis, University of Cambridge, 205 pp.

_ 1996: Rossby adjustment over a slope in a homogeneous fluid. J. Phys. Oceanogr., 26, 1646-1654.

Carnevale, G. F., and J. S. Frederiksen, 1987: Nonlinear stability and statistical mechanics of flow over topography. J. Fluid Mech., 175, 157-181.

_, R. C. Kloosterziel, and G. J. F. van Heijst, 1991: Propagation of barotropic vortices over topography in a rotating tank. $J$. Fluid Mech., 233, 119-139.

— , S. G. L. Smith, F. Crisciani, R. Purini, and R. Serravall, 1999: Bifurcation of a coastal current at an escarpment. J. Phys. Oceanogr., 29, 969-985. 
Gill, A. E., M. K. Davey, E. R. Johnson, and P. F. Linden, 1986: Rossby adjustment over a step. J. Mar. Res., 44, 713-738.

Hansen, B., and J. Meincke, 1979: Eddies and meanders in the Iceland-Faroe Ridge area. Deep-Sea Res., 26A, 1067-1082.

Johnson, E. R., 1985: Topographic waves and the evolution of coastal currents. J. Fluid Mech., 160, 499-509.

_ , and M. K. Davey, 1990: Free-surface adjustment and topographic waves in coastal currents. J. Fluid Mech., 219, 273 289.

Kinder, T. H., D. C. Chapman, and J. A. Whitehead, 1986: Westward intensification of the mean circulation on the Bering Sea shelf. J. Phys. Oceanogr., 16, 1217-1229.

Orlandi, P., 1990: Numerical simulation of vortices in presence of solid boundaries. Proceedings of the 8th GAMM Conference on Numerical Methods in Fluid Mechanics, P. Wesseling, Ed., Vieweg, 436-441.

Orlanski, I., 1976: A simple boundary condition for unbounded hyperbolic flows. J. Comput. Phys., 21, 251-269.

Poulain, P. M., 1999: Drifter observations of surface circulation in the Adriatic Sea between December 1994 and March 1996. J. Mar. Syst., 20, 231-253.
Salmon, R., G. Holloway, and M. C. Hendershott, 1976: The equilibrium statistical mechanics of simple quasi-geostrophic models. J. Fluid Mech., 75, 691-703.

Serravall, R., 1999: Dinamica di una corrente costiera in presenza di un gradino topografico. Ph.D. thesis, Università degli Studi di Roma "La Sapienza," 136 pp.

Spitz, Y. H., and D. Nof, 1991: Separation of boundary currents due to bottom topography. Deep-Sea Res., 38, 1-20.

Stern, M. E., and J. Austin, 1995: Entrainment of shelf water by a bifurcating continental boundary current. J. Phys. Oceanogr., 25, 3118-3131.

Willmott, A. J., and R. H. J. Grimshaw, 1991: The evolution of coastal currents over a wedge-shaped escarpment. Geophys. Astrophys. Fluid Dyn., 57, 19-48.

Zavala Sansón, L., and G. J. F. van Heijst, 2002: Ekman effects in a rotating flow over bottom topography. J. Fluid Mech., 471, $239-255$.

— — - and J. J. J. Doorschot, 1999: Reflection of barotropic vortices from a step-like topography. Nuovo Cimento, 22C, 909-929. 